\title{
TÉCNICA DE LICHTENSTEIN SOB ANESTESIA LOCAL EM HERNIORRAFIAS INGUINAIS
}

\author{
Lichtenstein technique under local anaesthesia in inguinal herniorrhaphies \\ João Eduardo Marques Tavares de Menezes ETTINGER ${ }^{1,2}$, Paulo Vicente dos SANTOS-FILHO², \\ Paulo Cézar Galvão do AMARAL ${ }^{1,2}$, Edvaldo FAHEL ${ }^{2}$
}

ABCDDV/575

\begin{abstract}
Ettinger JEMTM, Santos-Filho PV, Amaral PCG, Fahel E. Técnica de Lichtenstein sob anestesia local em herniorrafias inguinais. ABCD Arq Bras Cir Dig 2007;20(4):283-9

RESUMO - Racional - Herniorrafia inguinal, um procedimento cirúrgico comum no mundo todo, representa um importante gasto para saúde pública. Variadas abordagens cirúrgicas e opções de procedimentos anestésicos estão disponíveis para o reparo das hérnias inguinais atualmente. O objetivo do presente trabalho consiste em revisar e avaliar o tratamento cirúrgico inguinal pela técnica de Lichtenstein sob anestesia local. Métodos - Dados de pesquisa constituíram-se de artigos científicos publicados e disponibilizados no site do PubMed (www.pubmed.gov). A revisão de literatura baseou-se no cruzamento dos seguintes descritores: herniorrafia inguinal, anestesia local e técnica de Lichtenstein. Cento e oito publicações relacionadas ao tema foram revisadas. Conclusões - A combinação entre anestesia local e o emprego da técnica livre de tensão de Lichtenstein para o reparo de hérnia inguinal primária demonstrou resultados positivos. A associação descrita mostrou reduções significativas na recorrência de hérnia inguinal e nas complicações pós-operatórias, assim como diminuição da dor crônica eventualmente presente em uma avaliação tardia. O melhor controle da dor pós-operatória imediata, a alta hospitalar precoce e o retorno às atividades laborativas podem ser atribuídos de maneira direta ou indireta às técnicas empregadas, refletindo na satisfação do paciente e em baixos custos para saúde pública.

DESCRITORES - Herniorrafia inguinal. Anestesia local. Técnica de Lichtenstein.
\end{abstract}

\section{INTRODUÇÃO}

Anualmente são praticadas nos Estados Unidos da América cerca de 700.000 herniorrafias inguinais, gerando custos superiores a 2,8 bilhões de dólares ${ }^{26}$. Na Alemanha, cerca de 180.000 são diagnosticadas e tratadas anualmente $^{35}$. No Brasil, a realidade é a mesma. As hernioplastias inguinais foram responsáveis por 500 mil operações realizadas entre 1993 e 1996 pelos cirurgiões do Sistema Único de Saúde (SUS), o que representa 5,3\% de todas opeerações realizadas. Isto significou naquele período gasto para os cofres públicos de cerca de 100 milhões de reais ${ }^{25}$. Aliado a isso, as hérnias acometem freqüentemente indivíduos na faixa etária em que são mais produtivos e ativos fisicamente, não raro incapacitando-o para suas atividades rotineiras, gerando importantes implicações sócio-econômicas.A alta incidência dessa doença faz das hernioplastias as operações mais comumente realizadas por cirurgiões gerais ${ }^{35}$.

$\mathrm{O}$ tratamento da hérnia inguinal vem evoluindo bastante com o entendimento da anatomia, da fisiologia e, sobretudo da fisiopatologia. Os primeiros avanços significativos iniciaram-se com o advento dos materiais

Trabalho realizado no Departamento de Clínica Cirúrgica, Escola Bahiana de Medicina e Saúde Pública ${ }^{1}$ e Serviço de Cirurgia Geral, Hospital São Rafael², Salvador, Bahia, Brasil.

Endereço para correspondência: Paulo Vicente Filho, e-mail: paulovicentefilho@gmail.com de síntese e prótese. O estudo e o entendimento do comportamento ultra-estrutural das fibras de colágeno e de sua análise bioquímica também foram muito importantes para a evolução das técnicas atuais ${ }^{8}$. Grandes avanços no tratamento das hérnias inguinais são notados desde Bassini até o desenvolvimento da técnica sem tensão, preconizada por Lichtenstein ${ }^{23}$.

Atualmente há entre os cirurgiões e as diferentes escolas aonde eles se formam, preocupação e divergentes opiniões no que diz respeito às opções técnicas disponíveis para o tratamento das hérnias inguinais ${ }^{1}$. O Colégio Americano de Cirurgiões considera a técnica sem tensão de Lichtenstein o padrão-ouro no tratamento das hérnias inguinais primárias já que apresenta baixas taxas de recidivas e de complicações ${ }^{15}$.

Outro importante aspecto a ser discutido é o tipo de anestesia que deve ser utilizado ${ }^{1}$. As operações para correção da hérnia inguinal podem ser realizadas com anestesia local, regional (raqui ou peridural) ou geral. Dentre essas opções, a anestesia local parece ter melhor custobenefício embora no Brasil a maioria desses procedimentos é realizada com anestesia regional e mesmo em países como EUA, 70-90\% são realizadas com anestesia geral ou regional, cabendo as anestesias locais apenas 5-10\% das hernioplastias ${ }^{40}$. Os principais centros de referência no tratamento de hérnias inguinais utilizam anestesia local em mais de $95 \%$ dos casos, obtendo excelentes resultados ${ }^{40}$. Na Clínica Shouldice no Canadá, 95\% das hérnias inguinais 
são operadas com anestesia local, em experiência de mais de 290.000,00 operações ${ }^{7}$. No Instituto Lichtenstein, nos EUA, é rotina o uso de anestesia local ${ }^{4}$.

O presente estudo objetiva revisar e discutir a técnica de Lichtenstein sob anestesia local como tratamento das hérnias inguinais.

\section{MÉTODOS}

A revisão sistemática foi realizada entre dezembro e maio de 2005 nos bancos de dados mais importantes, no que concerne à indexação de publicações científicas: PubMed / Medline, Bireme, Scielo, Lilacs, além de livros, revistas, periódicos e sites especializados na Internet.

Todas as buscas basearam-se nas seguintes palavras-chave: "Hernia AND local AND anesthesia OR Lichtenstein AND local AND anesthesia OR Open AND laparoscopic AND hérnia." Os artigos perdidos na pesquisa inicial foram identificados a partir das referências dos artigos selecionados.

Foram excluídos artigos publicados em línguas que não fossem português, inglês e espanhol e artigos que não abordavam diretamente $\mathrm{o}$ assunto a ser revisado.

Foram selecionados 108 artigos de revisão de literatura, estudos prospectivos e relatos de casos, além de livros e sites médicos na internet. Os artigos identificados foram avaliados criticamente.

\section{REVISÃO DA LITERATURA}

\section{Técnica De Lichtenstein}

A descrição da técnica tension-free com uso de tela por Lichtenstein, há 20 anos, iniciou uma nova era nas operações das hérnias inguinais ${ }^{36}$. Ela foi descrita pela primeira vez em 1984 e evoluiu entre 1984 e 1988 para procedimento que, hoje em dia, é considerado o padrão-ouro para hernioplastias pelo Colégio Americano de Cirurgiões ${ }^{15}$ e por diversas outras instituições. A literatura mundial mostra que a técnica de Lichtenstein apresenta taxas de recidiva e complicações menores que $1 \%$, semelhantes entre cirurgiões experientes e cirurgiões inexperientes, já que os princípios básicos do procedimento, se respeitados, são fáceis de serem ensinados, de se aprender e principalmente de serem executados.

$\mathrm{O}$ atual papel da falha do metabolismo do colágeno na patogenia das hérnias inguinais tem levado à nova compreensão da patologia e das causas de insucessos nos procedimentos cirúrgicos ${ }^{34}$. $\mathrm{O}$ defeito do metabolismo do colágeno leva a enfraquecimento do tecido fibroconectivo e, por conseguinte, ao desenvolvimento das hérnias inguinais. A utilização desse tecido já danificado, sobretudo sob pressão, é contraproducente e vai de encontro aos princípios básicos da fisiologia, muitas vezes explicando o aparecimento de resultados insatisfatórios em algumas técnicas de hernioplastias.

Foi baseado nesse tipo de pensamento que cientistas buscaram alguma forma de contornar essa limitação. Para tal, lançaram mão do desenvolvimento de próteses para substituir a utilização de tecido danificado na hernioplastia. Os primeiros materiais sintéticos desenvolvidos foram associados a uma série de complicações, principalmente rejeição e infecção. Mais tarde, em meados da década de 50, Usher $^{39}$ popularizou o uso de tela de polipropileno com baixas taxas de rejeição e em 1984 (publicado em 1989) o grupo de Lichtenstein adotou o uso sistemático da tela nas cirurgias de hérnia inguinal, instituindo o termo "hernioplastia sem tensão".

Após alguns anos de análise da técnica, o próprio grupo de Lichtenstein e seus colaboradores observaram algumas falhas que foram corrigidas em 1996 e ainda neste mesmo ano padronizaram o tamanho $(16 \times 8 \mathrm{~cm})$ e a forma da tela ${ }^{2}$.

O princípio básico da técnica de Lichtenstein é promover hernioplastia sem tensão, não só presente durante a operação, na posição supina, mas como também no pósoperatório, em posição ortostática, evitando complicações decorrentes do aumento do gradiente de pressão intra-abdominal e do encolhimento da tela (ela encolhe $20 \%$ em ambas as direções depois da implantação) ${ }^{21}$. A conseqüência disso é mínima presença de dor no pós-operatório e baixas taxas de recidiva ${ }^{36}$.

Uma variedade muito grande de telas está à disposição dos cirurgiões. As propriedades ideais são resistência à infecção, permeabilidade molecular, transparência, integridade mecânica, flexibilidade, biocompatibilidade e ser inerte $^{18}$. Elas são classificadas em quatro tipos, de acordo com o tamanho dos poros. As do tipo I são aquelas com macroporos e incluem a tela monofilamentar que é mais popular em uso no momento ${ }^{18}$. O uso de telas de propileno com macroporos tem como vantagem permitir a presença de fibroblastos, macrófagos e fibras de colágeno no seu interior, além de favorecer angiogênese. Tudo isso resulta em melhor fixação da prótese na parede abdominal e melhores resultados ${ }^{36}$.

Na hernioplastia sem tensão, em vez de sutura com tensão das estruturas anatômicas - que não é fisiologicamente normal -, todo assoalho inguinal é reforçado com a inserção de uma tela. A prótese, que é posicionada entre o assoalho inguinal e a aponeurose do obliquo externo, estende-se além dos limites do triângulo de Hasselbach com o objetivo de promover sobreposição suficiente entre a tela e o tecido. Uma vez aumentada a pressão intra-abdominal, a contração do músculo oblíquo interno aplica contrapressão na tela que, desta forma, é utilizada a favor do próprio reparo.

O procedimento de Lichtenstein é tanto terapêutico quanto profilático e protege toda a região da virilha (inguinal) que é susceptível à herniação devido a defeitos mecânicos e a metabólicos.

A tela deve ser fixada cuidadosamente com uso de suturas de polipropileno ou sutura mecânica. Desta forma, a tela não dobra, não enruga, não se movimenta e nem enrola ao redor do cordão espermático, evitando recidiva e necessidade de reoperação ${ }^{36}$.

O método de Lichtenstein é simples e pode ser realizado por todos os cirurgiões, mesmo aqueles menos experientes 
ou sem interesse especial no tratamento das hérnias inguinais. É comprovadamente eficaz no que diz respeito à prevenção de recidiva e combina série de vantagens como simplicidade, efetividade, segurança e altas taxas de satisfação por parte dos pacientes ${ }^{36}$.

\section{Lichtenstein vs. outras técnicas}

Muitos são os artigos contidos na literatura que comparam os resultados da diferentes técnicas de hernioplastia. Atualmente, as principais comparadas pela literatura mundial são as de Lichtenstein e Shouldice.

Um dos principais aspectos comparados entre os tipos de operação é a taxa de recidiva. Quando se compara a tradicional técnica de Bassini com a de Lichtenstein fica clara a evolução das hernioplastias neste âmbito. Estudo retrospectivo realizado por Morkete M. et al. ${ }^{27}$ demonstrou o efeito da mudança de técnica de Bassini para Lichtenstein no protocolo de um hospital do Reino Unido em 1994. Os pacientes submetidos à técnica de Bassini em 1993 obtiveram 5\% de recidiva enquanto aqueles que em 1994 foram submetidos ao reparo sem tensão com uso de telas apresentaram taxas de 1,5\%. São descritos na técnica de Bassini índices de recidiva de até $15 \%$.

Os estudos mais atuais comparam os resultados da escola americana (Lichtenstein) com os da escola canadense (Shouldice). Em recente estudo retrospectivo, Aytac B. et al. ${ }^{5}$ compararam os resultados das duas técnicas em pacientes com hérnias unilaterais primárias. Estudou-se o tempo operatório, o de permanência hospitalar, necessidade de utilização de analgésicos no pós-operatório, complicações pós-operatórias, retorno ao trabalho e taxas de recidiva. Os autores concluíram que a técnica de Lichtenstein oferece menor tempo operatório, retorno mais precoce ao trabalho, menor necessidade de analgesia no pós-operatório e menor taxa de recidiva (Shouldice 4,1\% e Lichtenstein $0,8 \%)^{5}$.

As vantagens da técnica de Lichtenstein também são úteis para aqueles cirurgiões em treinamento. Danielsson P. et al. ${ }^{11}$ através de estudo prospectivo randomizado com 200 pacientes compararam os resultados das técnicas de Lichtenstein e Shouldice operadas por cirurgiões inexperientes. Concluíram que, para os aprendizes, a técnica de Lichtenstein é o melhor, já que se trata de técnica simples e com resultados pós-operatórios melhores. Não houve diferença significativa no que diz respeito ao tempo de duração da operação e presença de dor ou complicações no pós-operatório entre dois grupos ${ }^{11}$.

A comparação da taxa de recidiva, dificuldades técnicas e presença de dor crônica entre as técnicas de Shouldice e Lichtenstein são fundamentais para a determinação da técnica ideal. Em estudo prospectivo randomizado, Nordin P. et al. ${ }^{30}$ acompanharam 300 pacientes divididos em dois grupos (150 Lichtenstein e 150 Shouldice) desde o procedimento cirúrgico até três anos após. Como resultado, percebeu que a técnica de Lichtenstein era realizada em menor tempo e após 77 meses a taxa de recidiva foi de $4,6 \%$ no grupo de Shouldice contra $0,6 \%$ no grupo de Lichtenstein. Eles então concluíram que a técnica de
Lichtenstein é mais fácil de aprender, leva menos tempo e apresenta menor recidiva ${ }^{30}$.

Semelhante conclusão foi tirada por McGillicuddy J.E. ${ }^{24}$ em estudo prospectivo e randomizado realizado entre 1990 e 1995, quando 712 pacientes foram submetidos à hernioplastia e divididos igualmente em dois grupos (Lichtenstein e Shouldice). Para ele ambas as técnicas são comparáveis e efetivas, embora os resultados a longo termo favoreçam a sem tensão, já que apresenta menor recidiva, além de mais fácil de ser ensinada. A técnica de Shouldice pode ser útil nas hérnias estranguladas não femorais com necrose.

\section{Lichtenstein convencional vs. reparo laparoscópico}

As técnicas laparoscópicas para tratamento das hérnias já fazem parte da realidade atual. Existem atualmente três tipos de técnicas laparoscópicas para o tratamento das hérnias: IPOM (Intraperitoneal Onlay Mesh) que está em desuso, TEP (Totally Extraperitonial Repair) e TAPP (Transabdominal Preperitoneal Repair). Gokalp A. et al. ${ }^{17}$ realizaram estudo prospectivo randomizado comparando a técnica de Lichtenstein aberta e a laparoscópica TEP. Sessenta e dois pacientes foram operados pela técnica de Lichtenstein e 61 pela técnica laparoscópica extraperitoneal. Os pacientes foram acompanhados por dois anos e os resultados desse estudo não evidenciaram diferença significativa entre os grupos no que diz respeito à recidiva, dor pós-operatória, necessidade de analgésicos, complicações e tempo de hospitalização. Em contrapartida, a técnica laparoscópica foi em média 16 minutos mais demorada que a convencional e também foi considerada mais cara. O tempo de retorno ao trabalho foi menor naqueles que se submeteram à técnica laparoscópica. Os autores concluíram que a técnica convencional de Lichtenstein é superior no tratamento das hérnias inguinais primárias que a laparoscópica, e esta deve ser considerada no tratamento das hérnias recorrentes e bilaterais ${ }^{17}$. O uso da laparoscopia nas hérnias inguinais primárias ainda é controverso.

Alguns autores afirmam que o método laparoscópico sem tensão vem associado à baixas taxas de recidiva, menor dor no pós-operatório imediato e retorno mais precoce ao trabalho ${ }^{22}$. Entretanto, necessita de anestesia geral, é mais difícil de ser executada, necessita de curva de aprendizado maior e é mais freqüentemente associada a complicações intra-operatórias quando comparada ao método convencional aberto, incluído a técnica de Lichtenstein ${ }^{14}$.

Recente artigo publicado no The New England Journal of Medicine concluiu que o método convencional aberto é superior ao método laparoscópico seja pelas menores taxas de recidiva, seja pela maior satisfação dos pacientes. Nesse estudo, Neumayer et al. ${ }^{28}$ realizaram estudo prospectivo randomizado multicêntrico com 1696 pacientes que foram operados e acompanhados ao longo de dois anos. As taxas de recidiva das hérnias primárias operadas foram maiores no grupo da laparoscopia (10,1\% vs. 4,9\%). Entretanto, taxas semelhantes de recidiva foram encontradas nas hérnias recorrentes operadas. As complicações intra-operatórias e imediatas foram também maiores no grupo da laparoscopia 
(39\% vs. $33,4 \%$ ) embora complicações a longo prazo e mortalidade foram semelhantes nos dois grupos. O grupo dos pacientes submetidos a tratamento laparoscópico apresentou vantagens apenas no que diz respeito a menor dor no primeiro dia depois da operação e duas semanas depois dela. A partir de então, a dor foi se equilibrando e ao final de dois anos foi semelhante nos dois grupos ${ }^{28}$. Os pacientes operados pelo método aberto convencional retornaram as atividades normais em média um dia depois daqueles submetidos ao tratamento laparoscópico ${ }^{28}$.

Ettinger J.E. et al. ${ }^{13}$ encontram resultados semelhantes em sua casuística. Eles convergem quando afirmam que ambas as técnicas apresentam mesmas taxas de mortalidade. Também compartilham a conclusão que aqueles pacientes submetidos à laparoscopia apresentam menor dor no pósoperatório imediato e retorno mais precoce às atividades de rotina. Este mesmo estudo concluiu que os custos com a técnica laparoscópica são quase duas vezes maiores que com a técnica aberta de Lichtenstein. ${ }^{13}$.

Heikkinen T.J. et al. ${ }^{19}$, em estudo prospectivo e randomizado, compararam os custos entre herniorrafia laparoscópica e convencional aberta pela técnica de Lichtenstein, chegando também a conclusão que o método convencional é mais barato.

Estudo nacional, realizado por Brenner et al. ${ }^{8}$ revelou diferença não tão grande entre os custos da técnica aberta de Lichtenstein e o método laparoscópico TEP. Esse estudo prospectivo também concluiu que a operação aberta é mais rápida (com tempo médio de 102 minutos contra 127 minutos da laparoscópica). Após 30 dias, todos os pacientes de ambos os grupos referiram estar satisfeitos com o procedimento. Houve um caso de reoperação devido ao posicionamento errôneo da tela no grupo da laparoscopia extraperitoneal ${ }^{8}$.

Em estudo prospectivo e randomizado, Beets et al. ${ }^{6}$ acharam maior recidiva após reparo laparoscópico préperitoneal de hérnias inguinais recidivadas comparado com a aberta de Lichtenstein.

Resultados semelhantes com maior recidiva e maior número de complicações nos pacientes submetidos à hernioplastia por vídeo (TEP) comparadas com a técnica aberta de Lichtenstein, foram encontrados em outros estudos. A videocirurgia é indicada para correção de hérnias bilaterais, tendo como vantagem a utilização de pequenas incisões, menor dor no pós-operatório imediato e retorno mais rápido ao trabalho. Entretanto, devido a maior complexidade das operações endoscópicas, exige longo treinamento da equipe, maior tempo operatório e proporciona maior risco de lesão visceral e vascular ${ }^{8}$. Essa técnica deve ser reservada ao especialista ${ }^{8}$.

\section{Lichtenstein sob anestesia local}

Existe uma série de possibilidades de anestesia para esse procedimento. A escolha requer a avaliação da segurança para o paciente e rápido retorno para seu estado pré-operatório. Outras preocupações, como custos e satisfação, também devem ser consideradas. As principais modalidades anestésicas são infiltração local, bloqueio loco-regional, bloqueio subaracnóide, bloqueio epidural, bloqueio caudal e bloqueio paravertebral. As técnicas mais utilizadas são anestesia local, regional ou geral.

O uso de anestesia local não é novo. Flanagan e Bas$\operatorname{con}^{16}$ descreveram relatos feitos há mais de 100 anos sobre hérnias operadas com anestesia local por Harvey Cushing, usando cocaína ${ }^{16}$. Atualmente, o reparo das hérnias inguinais primárias com anestesia local é procedimento bem estabelecido ${ }^{3,9,16,26,37,38}$. Ele preenche os critérios ideais para o procedimento ambulatorial da hérnia inguinal: é seguro, simples e seu uso rotineiro já foi demonstrado como sendo eficiente na literatura ${ }^{3,12,20,32}$. A recuperação pós-operatória é definitivamente mais rápida após a anestesia loca ${ }^{40}$. Normalmente, pacientes submetidos a outros métodos anestésicos e cirúrgicos necessitam em média de pelo menos mais um dia de internação. A alta imediata e a deambulação precoce geram alto índice de satisfação para os pacientes ${ }^{40}$, diminuem a possibilidade de infecções pulmonares e cruzadas além de proporcionar menores custos para os hospitais, sobretudo os públicos que, na maioria dos casos, apresentam dificuldades financeiras. Com essa técnica, usa-se a sala de cirurgia por menos tempo, ocupa-se menos os leitos hospitalares e menos gastos para os sistemas de saúde. Recente estudo prospectivo e randomizado conduzido por Gonullu et al. ${ }^{20}$ comparou os custos de herniorrafias inguinais pela técnica de Lichtenstein com anestesia local e anestesia geral. Os resultados evidenciaram que o procedimento com anestesia geral foi mais caro do que a com anestesia local. Outros estudos prospectivos compartilham da mesma conclusão ${ }^{37}$.

De uma forma geral, os procedimentos anestésicos utilizados para hernioplastias inguinais apresentam baixa morbidade. No entanto, a anestesia geral e a raquidiana estão mais comumente associadas a disfunções gastrointestinais tais como náuseas e vômitos, retenções urinárias, complicações cardíacas, hipotensão postural e maiores índices de tromboflebite, o que não vem sendo demonstrado com uso de anestesia local ${ }^{40}$. $\mathrm{O}$ fato da anestesia envolver apenas uma área limitada faz com que ela não interfira no funcionamento de outros órgãos e promova relaxamento muscular localizado, afetando menos a ventilação. Gonullu et al. ${ }^{20}$ também compararam a função pulmonar após herniorrafia sob anestesia local e geral. Em ambos os métodos anestésicos a função pulmonar foi afetada, embora os pacientes que se submeteram a anestesia local obtiveram significativamente melhor função pulmonar.

Diversos outros importantes estudos comprovam a superioridade da anestesia local em relação às demais técnicas anestésicas. Amid et al. ${ }^{3}$ em série de 12 mil pacientes operados sob anestesia local no Instituto Lichtenstein, afirmam tratar-se de método seguro, simples, efetivo e econômico. Kark A.E. et al. ${ }^{20}$ em estudo prospectivo randomizado com 2906 pacientes e seguimento de 18 meses a cinco anos, descrevem que os benefícios econômicos da anestesia local são decorrentes da baixa morbidade, rápido retorno ao trabalho e baixas taxas de recidiva. Ozgun et al. ${ }^{32}$ em estudo prospectivo e randomizado, concluem que 
a anestesia local é totalmente plausível como procedimento ambulatorial, já que há menor incidência de náuseas e retenção urinária, menor necessidade de analgesia no pós-operatório, menor tempo operatório e maior satisfação dos pacientes, comparada com a anestesia espinhal e geral. Essa mesma conclusão foi tirada por Valente et.al ${ }^{23}$ em estudo nacional prospectivo e publicado recentemente na Acta Cirúrgica Brasileira. Em estudo retrospectivo, Subramanian P. et al..$^{38}$ evidenciaram que apenas um dos 56 pacientes operados com anestesia local $(2 \%)$ necessitou de mais de 24 horas de analgesia comparado a quatro dos $37(11 \%)$ no grupo da anestesia geral. Dados semelhantes foram encontrados por Espinoza et al. ${ }^{12}$ com grupo de pacientes operados com anestesia local que apresentou menor dor no pós-operatório comparado ao grupo operado com anestesia espinhal. Callesen Torben et al. ${ }^{9}$ ressaltam que o procedimento é seguro, possui poucas complicações pósoperatórias e apresenta aceitável satisfação, embora a dor intra-operatória tenha aparecido como fator de insatisfação em 13\% dos pacientes avaliados. Dados semelhantes foram encontrados por Pritchard et al. ${ }^{33}$ em estudo prospectivo onde evidenciaram que $23 \%$ dos pacientes operados sob anestesia local queixaram-se de dor leve durante o procedimento. Nordin Par et al. ${ }^{29} \mathrm{em}$ recente estudo prospectivo, randomizado e multicêntrico publicado no The Lancet, compararam os resultados de 616 herniorrafias operadas sob anestesia local, regional e geral. Concluíram que a anestesia local apresenta menor dor no pós-operatório imediato, embora no $8^{\circ} \mathrm{e} 30^{\circ}$ dia do seguimento, não houve diferença significativa entre os três grupos. Nesse mesmo estudo, evidenciaram que a operação com anestesia local (da indução anestésica ao fim do procedimento) é mais rápida, o tempo de internação é menor e há menor número de complicações, sobretudo dificuldades miccionais. Outros estudos prospectivos randomizados compararam os métodos anestésicos na herniorrafia inguinal. Todos eles também demonstram as vantagens acima citadas da anestesia local em pacientes selecionados. Quatro deles evidenciaram menor dor pós-operatória na anestesia local ${ }^{37}$.

Callensen et al..$^{10}$ operaram 995 pacientes com anestesia local, 961 deles receberam alta em média 95 minutos após o procedimento. Outros autores obtiveram, em 446 pacientes operados, tempo médio de internação de apenas 85 minutos. Equipes inexperientes não conseguem obter períodos de internação tão rápidos, embora ainda sejam menores que 24 horas.

O'Dwyer P.J. et al. ${ }^{31}$ não encontraram diferença significativa entre os resultados de herniorrafias usando anestesia local e geral. Eles avaliaram em 276 pacientes a dor pós-operatória, o tempo de retorno ao trabalho e os custos. Para os autores, devem ser oferecidas ao paciente as duas possibilidades de anestesia ${ }^{31}$.

Em estudo nacional, conduzido por Edgar Valente et al. 93,3\% dos pacientes deambularam precocemente após herniorrafia inguinal sob anestesia local.

A anestesia local é contra-indicada no caso de pacientes psiquiátricos, pacientes que se recusam a cooperar, aqueles que possuem grandes hérnias encarceradas ou estrangula- das, hérnias inguinoescrotais e pacientes obesos ${ }^{35}$.

Outras restrições ao método descritas na literatura são: ardência, dor na infiltração dos anestésicos, pacientes obesos, hérnias encarceradas, estranguladas e inguinoescrotais $^{35}$. Alguns autores defendem que a anestesia local em crianças seja algo difícil de ser realizado embora Minossi et al. ${ }^{26}$ concluíssem que a anestesia local associada à sedação em crianças é procedimento simples e seguro. Outros julgam que este procedimento possua restrições em relação às hérnias recidivadas ${ }^{35}$. Isso não foi demonstrado em estudo realizado por Callesen et al. ${ }^{9}$. Eles concluíram que hérnias recidivadas podem ser seguramente operadas sob anestesia local, mantendo sempre cuidado especial em relação à possibilidade de dor intra-operatória

\section{Técnica de anestesia local}

Como anestésico local, é utilizada mistura de cloridrato de lidocaína sem vasoconstrictor a $1 \% 1,50$ ou $2 \%$ $(20 \mathrm{~mL})$, cloridrato de bupivacaína com vasoconstrictor a $0,5 \%(20 \mathrm{~mL})$ e água destilada $(10 \mathrm{~mL})$. Para cada 9 $\mathrm{mL}$ da solução, adiciona-se $1 \mathrm{~mL}$ de bicarbonato de sódio com intuito de manter o $\mathrm{pH}$ em torno de 71,50. Nordin Par et al. utilizaram mistura 50:50 de mepivacaína a 1\% e bupivacaína a $0,5 \%$.

A técnica em leque empregada para anestesia local inicia-se ao infiltrar $5 \mathrm{~mL}$ da solução em "leque", dois dedos medial e superiormente à crista ilíaca ântero-superior e em profundidade, visando atingir o plano entre o músculo transverso e o oblíquo interno e desta forma bloqueando os nervos íleo-hipogástrico e íleo-inguinal. Depois se infiltra mais $5 \mathrm{~mL}$ da solução em "leque" na projeção do tubérculo púbico; após afastamento lateral do funículo espermático, a agulha penetra até encontrar o periósteo. Por fim, infiltração da pele e da tela subcutânea ao longo da arcada inguinal com 10 a $15 \mathrm{~mL}$ da solução. Durante a execução da operação, promove-se a infiltração do anel profundo com $5 \mathrm{~mL}$ da solução; no caso de hérnias indiretas, a base do saco herniário é anestesiada, uma vez que sua tração é dolorosa.

A sedação do paciente mostra-se eficaz para diminuir ansiedade, proporcionar conforto e aumentar a tolerância ao método ${ }^{10,23}$. Ela normalmente é feita com Midazolan, 101,50 a 3084,98 minutos antes da operação, na dose de $0,05 \mathrm{mg}$ a $0,3 \mathrm{mg}$ por quilo de peso, reduzindo essa dosagem para metade em pacientes com menos de 60 anos $^{23}$. Pode-se também utilizar benzodiazepínicos, barbitúricos de curta duração, antieméticos e analgésicos potentes.

A complicação mais importante da infiltração local é a intoxicação sistêmica que pode ser prevenida ao evitar injeção intravascular e ao administrar sempre a dose correta.

Os principais agentes utilizados na anestesia local são a Lidocaína a $0,5 \%$ e a Bupivacaína a $0,125 \%$ e $0,25 \%$. A associação desses dois anestésicos visa encurtar o tempo de latência (efeito da lidocaína) e prolongar o efeito da anestesia por 4 a 6 horas (bupivacaína), o que é suficiente não só para concluir o ato cirúrgico, mas como também 
para permitir analgesia pós-operatória. Quando a solução anestésica não contém a bupivacaína, pode-se proceder, ao fim do procedimento nova infiltração em cada camada com a mesma solução, com o intuito de proporcionar ausência de dor nas primeiras 4 a 6 horas do pós-operatório. Na região inguinal, por se tratar de extensa área a ser anestesiada, é preferível a utilização de anestésicos diluídos a baixas concentrações. Deve ser acrescentado $0,1 \mathrm{~mL}$ de adrenalina a 1:1000 para cada $20 \mathrm{~mL}$ de solução anestésica. Vale lembrar que soluções previamente preparadas têm maior custo e pH mais ácido para conservar a adrenalina, o que aumenta o tempo necessário para ser iniciado o efeito anestésico. Alguns autores contra-indicam o uso de vasoconstritores, pois eles estariam relacionados a maior número de hematomas no pós-operatório. $\mathrm{O}$ uso do bicarbonato diminui a dor na indução anestésica.

Outra técnica utilizada é a de Harvey-Cushing. Seu aspecto mais importante é o bloqueio dos nervos íleohipogástrico e íleo-inguinal, já que estes são responsáveis pela inervação das principais estruturas da área operada. Suas principais complicações são a inquietação e irritação (deve ser tratada com midazolan, diazepam ou tiopental mais O2 sob máscara), convulsões (também tratadas da mesma maneira) e colapso cardiovascular: Um cuidado que deve ser tomado durante o bloqueio anestésico é que depósito em local muito próximo ao ligamento inguinal, inferiormente à espinha ilíaca e em grandes quantidades, pode resultar em bloqueio temporário do nervo femoral, com paralisia do músculo quadríceps e conseqüentemente retardar um dos grandes trunfos da anestesia local que é a deambulação precoce.

Durante o procedimento, os pacientes devem ser mantidos com monitorização eletrocardiográfica, pressórica e oximétrica.

\section{Considerações finais}

A possibilidade de se realizar herniorrafias inguinais em regime ambulatorial traz também como vantagem o aprendizado acadêmico. Hospitais-escola devem se beneficiar dessa modalidade cirúrgica uma vez que mais operações são realizadas em menor intervalo de tempo, implicando em maior aprendizado e melhor formação dos futuros cirurgiões. Da mesma forma, devido à escassez das vagas nos hospitais públicos, mais pacientes podem ser operados evitando filas de espera e transferências para outras instituições.

Alguns detalhes devem ser enfatizados a local. Inicialmente, o cirurgião deve orientar o paciente quanto à técnica que será empregada. Desta forma, ele não é surpreendido e obrigado a vivenciar experiência desagradável no intraoperatório, caso ele venha a acordar da sedação ou sentir dor. Deve também sempre ter em mente que a manipulação de estruturas profundas precisa ser cuidadosa, já que a anestesia é local.

Alguns cirurgiões não apreciam a infiltração local com anestésicos tendo em vista que tal procedimento promova distorção da anatomia local. Outros advogam que tal método prejudica o relaxamento muscular e conseqüentemente a técnica operatória. Ambas as objeções são válidas, porém na prática isto não acontece e os benefícios da técnica mostram-se muito maiores.

Outro ponto passível de discussão é a utilização de antibioticoprofilaxia. Alguns autores são contra e outros a favor. Isso, devido aos diferentes resultados encontrados nos estudos disponíveis na literatura. Quando empregada, pode ser feita com cefalosporinas de $1^{\mathrm{a}}$ ou $2^{\mathrm{a}}$ geração.

Apesar de todas as vantagens já discutidas, a técnica de Lichtenstein sob anestesia local não é amplamente utilizada em nosso meio. Pelo contrário, poucos são os centros aonde essa modalidade cirúrgica é praticada. Recentemente quatro trabalhos científicos nacionais sobre tratamento cirúrgico de hérnia inguinal foram publicados e em nenhum deles a anestesia empregada foi local ${ }^{25}$. Mas não é só no Brasil que isso acontece. Dados obtidos a partir de longos estudos epidemiológicos realizados em países desenvolvidos como Dinamarca, Escócia, Suécia e Inglaterra evidenciaram que apenas de $2 \%$ a $15 \%$ das hérnias inguinais operadas eram feitas com anestesia local.

\section{CONCLUSÃO}

A técnica de Lichtenstein empregada sob anestesia local é método simples, facilmente reprodutível, seguro, prático, econômico e apresenta bons resultados com baixos índices de recidiva e altos índices de satisfação para os pacientes previamente selecionados, apesar de pouco executada sob anestesia local no Brasil e em diversos outros países. 
Ettinger JEMTM, Santos-Filho PV, Amaral PCG, Fahel E. Lichtenstein technique under local anaesthesia in inguinal herniorrhaphies. ABCD Arq Bras Cir Dig 2007;20(4):283-9

ABSTRACT - Background - Inguinal herniorrhaphy, a common surgical procedure performed worldwide, represents an important expense for public health. A variety of surgical approaches and options for anesthesical procedures are available nowadays for inguinal hernia repair. The aim of this study is to review and to evaluate the Lichtenstein inguinal surgical treatment under local anesthesia. Methods - Researching data consisted of scientific articles published and available at PubMed site (www.pubmed.gov). Literature review based on crossing the following headings: inguinal herniorrhaphy, local anesthesia and Lichtenstein technique. One hundred and eight publications related to the subject were reviewed. Conclusion - Combination between local anesthesia and tension-free Lichtenstein technique for primary inguinal hernia repair demonstrates positive results. The association described shows a significantly decrease in inguinal hernia recurrence and in postoperative complications, as well as reducing chronic pain that might be present in a late follow-up. The better immediate postoperative pain control, early hospital patient discharge and a faster return to job activities may all be attributed directly or indirectly to the technique implemented, reflecting increased patient satisfaction and lower costs for public health.

HEADINGS - Inguinal herniorrhaphy. Local anesthesia. Lichtenstein technique.

\section{REFERÊNCIAS}

1. Amado WJ. Anestesia na cirurgia para hérnia. Clin Surg Am North 1993; $3: 449-462$

2. Amid PK, Shulman AG. Tension-free repair of inguinal and aponeurotic hernia. G Chir 1993; 14:145-154

3. Amid PK, Shulman AG, Lichtenstein IL. Local anaesthesia for inguinal hernia repair step-by-step procedure. Ann Surg 1994; 220:735-737.

4. Amid PK, Friis E, Horeyseck KM. A Multi-center experience with 6,764 Lichtenstein tension-free hernioplastias. Hernia1999; 3:47-48

5. Aytac B, Cakar KS, Karamercam A. Comparison of Shouldice and Lichtenstein repair for treatment of primary inguinal hernia. Acta Chir Belg 2004 Aug; 104(4):418-21

6. Beets GL, Dirksen CD, Go PM, Greisler FE, Baeten CG, Kootstra G. Open or laparoscopic preperitoneal mesh repair for recurrent inguinal hernia? A randomized controlled trial. Surg Endosc 1999; 13:323-327

7. Bendavid R. Results of open techniques of hernia repair. In: Schumpelick, V.; Wants, G.E. Inguinal hernia repair. 1ed. St. Moritz: Basel Karger; 1995. p 379-93

8. Brenner AS, Widerkehr J, Brenner S. Estudo comparativo entre herniorrafias Inguinais Lichtenstein e Videocirurgia Extraperitoneal Sem Sutura : Custos e Resultados Imediatos. Revista Brasileira de Videocirurgia 2004; 2(2): 63-67

9. Callesen T, Bech K, Kehlet H. One-thousand consecutive inguinal hernia repairs under unmonitored local anaesthesia. Anesth Analg 2001 Dec;93(6):1373-6, table of contents

10. Callesen T, Kehlet H. Inguinal herniotomy. Which kind of anesthesia ? Economical considerations. Ugeskr Laeger 1995; 157: 421-424.

11. Danielson P, Isacson S, Hansen MV. Randomised study of Lichtenstein compared with Shouldice inguinal hernia repair by surgeons in training. Eur J Surg 1999 Jan; 165(1):49-53

12. De La Cuadra Espinoza R, Volosky L, Brunet L, Silva JJ. Anestesia local vs anestesia espinal en hernioplastia inguinal: comparación de efecto anestésico postoperatorio / Local anestesia vs spinal anestesia in inguinal hernioplasty: comparison of the postsurgical anesthetic effect. Rev chil cir 2002; 54(6):649653, tab

13. Ettinger JE, Fahel E, Amaral PC. Retrospective, comparative study between the Videolaparoscopic (TAPP) and Lichtenstein techniques for inguinal hernia repair. Ciência e Saúde, Revista Científica do Hospital São Rafael 2003; 1:1721.

14. Fitzgibbons RJ, Campos J, Cornet DA. Laparoscopic inguinal herniorrhaphy: results of a multicenter trial. Ann Surg 1995; 221:3-13

15. Fitzgibon R. Management of an inguinal hernia: Conventional? Tension-free? Laparoscopic? Or maybe no treatment at all. General Sessions of the American College of Surgeons. 2000; 86 Annual Clinical Congress

16. Flanagan Jr. L, Bascon JU. Correção da hérnia inguinal: Abordagem do paciente externo com anestesia local. Clin Cirur Am Norte 1984; 2:271-282.

17. Gokalp A, Inal M, Maralcan G, Baskonus I. A prospective randomized study of Lichtenstein open tension-free versus laparoscopic totally extraperitoneal techniques for inguinal hernia repair. Acta Chir Belg 2003 Oct; 103(5): 502 506

18. Goldstein HS. Selecting the right mesh. Hernia 1999; 3:23-26 .

19. Heikkinen TJ, Haukipuro K, Hulkko A. A cost and outcome comparison between laparoscopic and Lichtenstein hernia operation in a day-case unit. A randomized prospective study. Surg Endosc 1998; 12:1199-1203
20. Kark AE, Kurzer MN, Belsham PA. Three thousand one hundred seventy-five primary inguinal hernia repairs: advantages of ambulatory open mesh repair using local anesthesia. J Am Coll Surg 1998 Apr;186(4):447-55; discussion 456.

21. Klinge U, Muller M. Shrinking of polypropylene mesh in vivo: Na experimental study in dogs. Eur J Surg 1998; 164:965

22. Liem MSL, Van Der Graff V. Comparison of conventional anterior surgery and laparoscopic surgery for inguinal hernia repair. New England Journal of Medicine 1997;336:1541-7

23. Lima Neto EV, Goldenberg A, Juca MJ. Resultados imediatos da herniorrafia inguinal com anestesia local associada com sedação. Acta Cirúrgica brasileira 2003; 18(5) 478-484

24. McGillicuddy JE. Prospective randomized comparison of the Shouldice and Lichtenstein hernia repair procedures. Arch Surg 1998Sep; 133(9):974-978

25. Melo RM, Codazi AO, Matias IS. Reparo pela técnica de Shouldice modificada nas hérnias inguinais primárias. Rev CBC 1998; 25(3): 167-170

26. Minossi JG, Pedro FAG, Vendites S. Herniorrafia inguinal com anestesia local. Arq Gastroenterolog 1992; 29:18-22.

27. Mokete M, Earnshaw JJ. Evolution of an inguinal hernia surgery practice. Postgrad Med J 2001; 77:188-190

28. Neumayer L, Jonassom O, Gibbs J. Open Mesh versus Laparoscopic mesh repair of inguinal hernia. The New England Journal of Medicine 2004; 350(18): 1819-27

29. Nordin P. Local, regional, or general anaesthesia in groin repair : multicenter randomized trial. The Lancet 2003; 13: 853-857

30. Nordin P, Jansson C, Svensson C, Edlund G. Randomized trial of Lichtenstein versus Shouldice hernia repair in general surgical practice. Br J Surg 2002 Jan; 89(1):45-49

31. O’Dwyer PJ, Serpel MG, Millar K, Young D. Local or general anaesthesia for open hernia repair: a randomized trial. Ann Surg 2003; 237(4): 574-9

32. Ozgun H, Kurt MN, Kurt I, Cevikel MH. Comparison of local, spinal, and general anaesthesia for inguinal herniorrhaphy. Eur J Surg 2002;168(8-9):4559.

33. Pritchard TJ, Bloom AD, Zollinger RM. Pitfalls in ambulatory treatment of inguinal hernia in adults. Sur Clin North Am 1991; 71:1353-62.

34. Rosch R, Klinge U, Schumpelick V. A role of the collagen I/III and MMP-1/-13 genes in primary inguinal hernia? BMC Med Genet 2002; 3:2

35. Rutkowi M, Robbins AW. Aspectos demográficos classificatórios e sócioeconômicos do reparo herniário nos Estados Unidos da América. Cli Cirur Am Norte 1993; 3: 433-38

36. Sakorafas GH, Halikias I, Nissotakis C, Stavrou A, Kassaras GA. Open tension free repair of inguinal hernias; the Lichtenstein technic. BMC Surgery 2001; $1: 3$

37. Song D, Greilich NB, White PF. Recovery profiles and costs of anaesthesia for outpatient unilateral inguinal herniorrhaphy. Anesth Analg 2000; 91:876-881

38. Subramaniam P, Leslie J, Gourlay C, Clezy JK. Inguinal hernia repair : a comparison between local and general anaesthesia. Aust N Z J Surg 1998; 68 : 799-800

39. Usher FC, Allen JE, Crosthwait RW, Cogan JE. Polypropylene monofilament. A new, biologic inert suture for closing contaminated wounds. JAMA 1962; 179:780

40. White PF. Optimizing anaesthesia for inguinal herniorrhaphy: General, Regional or Local Anaesthesia? Anesth Analg 2001; 93:1367-1369

Conflito de interesse: não há

Fonte financiadora: não há

Recebido para publicação em: 30/06/2007 Aceito para publicação em: 23/09/2007 